\title{
Performance Analysis of Peer to Peer Video on Demand System using V-Chaining Mechanism
}

\author{
Hareesh.K \\ Research scholar \\ Jawaharlal Nehru Technological University, \\ Anantapur, A.P India
}

\author{
Manjaiah D.H \\ Professor \& Chairman \\ Department of Computer Sciences, \\ Mangalore University, Mangalore
}

\begin{abstract}
Video on demand is a popular multimedia application on the internet. Now a day's peer to peer video on demand is the most popular technique that captures most of the users on the internet. Therefore, we have proposed a video on demand based on the peer to peer network and also defined a detailed working principle of peer to peer video on demand using chaining mechanism. In comparison to it, we have also considered some of the existing approaches that use chaining mechanism for the transmission of video streams for the video on demand system. To our proposed video on demand system architecture, we have proposed Video Chaining (V-Chaining) mechanism that enhances the performance of the chaining mechanism in terms of bandwidth, buffer in both server and peer load. Hence, we have derived an analytical model for different performance metrics for our mechanism and the same is simulated to have fairer comparisons between the existing mechanism and with our mechanism. The result of the simulation shows that our mechanism fairs better in all aspects of performance metrics than the existing mechanism such as standard, advanced, optimal and accelerated chaining.
\end{abstract}

\section{Keywords}

Chaining, peer, bandwidth, buffer, VoD, video

\section{INTRODUCTION}

The multimedia applications are the most commonly used in human day to day life. The several applications of VoD systems are the digitally transmitted movies, live streaming of video's, distance learning, TV programs etc., The large number of concurrent users over the internet can be watch the favorite movies and downloads the videos can be observed in everyday. Video streaming supports large number of concurrent users compared to other applications available on the Internet, which can consume more network bandwidth while streaming the videos. We can download the any videos from the remotely available media server using the following three approaches. Firstly offline streaming, the prerecorded videos are streamed completely into the users peer before they play out the favorite movies. Secondly live streaming, the media server starts broadcast the live movies, programmes to the subscribed users who is having an authentication to watch immediately starts streaming using his/her authentication to watch, streaming and broadcast the video streams to the adjacent peers. Some examples are cool streaming [1][2] etc. Thirdly Video on Demand (VoD) system; prerecorded movies streamed to the requested users, who can start watching at the instantly they can choose the favorite movies and may have streaming/download and may not forward the video streams to the adjacent peers which is called as selfish peers and also having the VCR controls like fast forward, pause, stop, and fast backward operations may perform for the favorite movies. Now a day, the VoD system is become more popular service, which will serve the global users over the internet. The media server maintains the video directory having the video frames, title, movieID, popularity, timestamps and Quality of Service for the streamed/streaming movies. VoD system architecture is one of the traditional client/server architecture. The client is always request the favorite movie title to the media server. If the requested movie is available, then media server starts streaming the movie to the peer for playback. The number of peer increases, server bandwidth and load on the server gradually increases. To overcome this problem, multiple servers such as parallel and distributed servers are placed in the region based to serve the group of users rather than serve all the users in the system. To upgrade the media server normally the cost will be more. But to handle the global users requests requires high end server like i) Content Delivery Network (CDN) ii) Proxy based system iii) Peer to Peer systems. In CDN [4] [5] the users can caches the video streams from the Point of Presence (PoP) of ISP's that stores the video in the subscriber peer This approach is not cost effective but reduces load on the server. To cache the video requires an additional hardware and a software requirement is one of the major disadvantages. In proxy based approach [6] [7] the media server caches the video streams to the proxy server. This approach is not scalable, but cost effective and reduces the load on the server. The video streaming in peer to peer architecture [8] [9] [10] is always relies on the application protocol. In this architecture, the peers act as miniature server can upload or download the video streams to the neigh boring peers and this streamed/streaming information in turn send to the video server. This architecture can reduce the load on server by sharing the network bandwidth among the peers. It is also more scalable and supports millions of global users; however, all the user peers are not involved in video streaming is not fair among the peers. To further reduce the load on the server of the VoD system, earthworm approach is proposed [11] to cache the video streams to the nearer peer. In this approach, the users of the peer can play back the video streams to the neighboring peers with adequate buffer delay which is called as basic chaining. This approach further enhanced as standard, advanced, and optimal chaining [6][14][15] approaches. These approaches usages more uplink bandwidth and buffer both in peer side as well as server side. Eventually we can observe that, users can play out the videos but the majority of the peers will stop forwarding video stream once finishes by playing the video. Now, the requested peer will starts receive the video streams from the neighboring peer. The peer will receive missing parts from the server dramatically increases the load on the server. Accelerated chaining [13] addresses these concern by requiring peers to forward their video streams at a rate slightly higher than their video consumption rate have a dramatic impact on the server load. However demand for digital quality videos are expected even for the MPEG-2 videos. For such applications the existing chaining approaches will not scalable to meet in sharing of resources like bandwidth and buffer among the 
peers over the network. For this overall performance of VoD system will degrade and occurrences of same data will commonly found while streaming among the peers. Hence we proposed Video Chaining (V-Chaining) mechanism compared with existing approaches, which will improve the overall system performance. The rest of the paper is organized as follows: Section 2 reviews some previous related works; Section 3 presents an overview of the proposed VoD architecture; Section 4 presents the chaining mechanism; Section 5 presents proposed V-Chaining algorithm; Section 6 evaluates the performance analysis; Section 7 presents simulation and results; Section 8 concludes the paper.

\section{RELATED WORK}

In this section presents the brief discussions of the previous research works in the various chaining approaches of video on demand system.

\subsection{Standard Chaining}

In standard chaining [6] chaining between each peer happens that, the first peer in the chain receive the video streams from the server and subsequent peers in the chain receive their video stream from their neighboring peers, which are in the chaining. As a result of this, we have observed some of the disadvantages that, the video streams are pipelined through peers belonging to the same chain. Here a new chain has to be restarted every time the inter arrival between the two successive peers requests exceed the buffer capacity of the previous peer buffer. Since, the chaining among the peers requires small buffers in the peers. In our scheme video streams are not pipelined through the peers belonging to same chain. A new chain has not restarted every time the inter arrival between the two successive peers exceed the buffer capacity of the previous peer buffer. The user can get the video stream from the neighboring peers without any duplication.

\subsection{Advanced Chaining}

The main disadvantages of advanced chaining [14] in its poor performance observed in the inter arrival rates of the requests of peer. More specifically, whenever the time interval between two consecutive requests among the peers exceed time of the video time in minutes. In this chaining we have studied that, every inter arrival of requests exceed the duration of video, to overcome the above problem by inserting every time in minutes the idle peers, and these idle peers will delay the video stream among the peers. In our proposed mechanism, we have not inserting idle peers, the differences of inter arrival of requests will not exceed the duration of video, and in turn our mechanism will not delay the video streams once each peer upload the video stream to the requested peer our proposed chaining algorithm checks certain conditions discussed in section 4 . If the condition is satisfied the peer will starts streaming the video to the requested peer.

\subsection{Optimal Chaining}

This chaining [12] [15] also managing all the peers buffer's as a single shared resource. As we have noticed as a result of this, peers can borrow the buffers from the neighboring peers in order to bridge the gaps between the incoming requests of several peers. In this mechanism also introduced the streaming proxy servers in order to increase the chaining responses and resilience. In optimal chaining approach, if a failure occurs on a chain single shared resource causes entire system will fail instead in our scheme we use multi shared resources.

\subsection{Expanded chaining}

Expanded chaining is also known as cooperative video distribution protocol [16] it also improve the chaining by taking the advantage of large buffer size of all the peers in the chaining. In this mechanism the main drawback that, the peer will disconnect also stop forwarding the video stream once they have finished the playback of the video. But in standard chaining, each peer forwards the video stream to the next immediate peers starts sending the beginning of the video streams, when a peer has finished playing the video the chain will disconnect and also stop transmitting the video stream. But the streaming server will transmit the remaining parts of the video. In our scheme we are not using the larger buffer size while involved chaining among all the peers, instead we have used the peer buffer based on the streaming time line of the video.

\subsection{Accelerated Chaining}

In case of standard and advanced chaining completely eliminate the server workload that the peer will always keep forwarding the video stream the next neighboring peers in the chain once they have finished playing the video. In above mechanism assumption is not reasonable because the most of the peers are disconnect once they played the video and most of the significant number of peers will also disconnect without playing the full length of video. In accelerated chaining [13] peers will forwards the video stream to the neighboring peers in the chain at slightly higher rate compared to other chaining mechanisms and on the video consumption rate. In accelerated chaining higher rate forwarding the video stream overflows the buffer due to this, we have forwarding the video stream based on the availability of bandwidth and playback rate.

\section{VoD SYSTEM ARCHITECTURE}

The peer to peer video on demand (P2P VoD) system architecture consists of a media server, proxy servers and peers in a cluster. The media server contains movie database in which there will be a collection of video data files. The video data file's information such as index, popularity, minimum bandwidth requirement and minimum buffer capabilities are also stored in the media server. Proxy server in this architecture is used to cache video data files for the nearer peers in a cluster. The purpose of proxy server is to reduce the load on the multimedia server by caching the video data files. A cluster is a logical connectivity of peers which is headed by a proxy server. A peer can be a seed peer or a non-seed peer. A seed peer has sufficient bandwidth and buffer capacity as well as it can store and forward video data files to other peers. A non seed peer is a user's peer, which has only required configuration and it can only playback the received video data file and cannot store or forward the video data files to other peers.

\subsection{Working Principle}

Initially, suppose if a peer makes a request to the media server, and then the media server downloads the entire video data files to the nearest proxy server of the requesting peer. In the first case, it is assumed that none of the peers are requested for the same movie. Thereby, after downloading movie to the proxy server, the proxy server will transmit the movie to the requesting peer. Subsequently, if another peer from the same cluster makes a request for the same movie to the media server. Then the media server looks in to its current streaming movies database for the nearest proxy server and its availability of the movies. If such entry is found then server redirects the requesting peer to the nearest proxy server. Again the proxy server applies the same procedure to find out 
that any of the peers in the cluster has the movie in its buffer. If such peer is found, then the requesting peer will be redirected to that peer has the same movie. Then on the transmission occurs from that peer to the requesting peer. The transmission of video data file from that of peer to another peer is called chaining.
However, if entry is not found in the proxy server, then the proxy server starts transmitting the video data files to the requesting peer. Elsewhere, if the entry is not found in the server then the procedure is followed as if it is a first request from the cluster However, if entry is not found in the proxy server, then the proxy server starts transmitting the video data files to the requesting peer. Elsewhere, if the entry is not

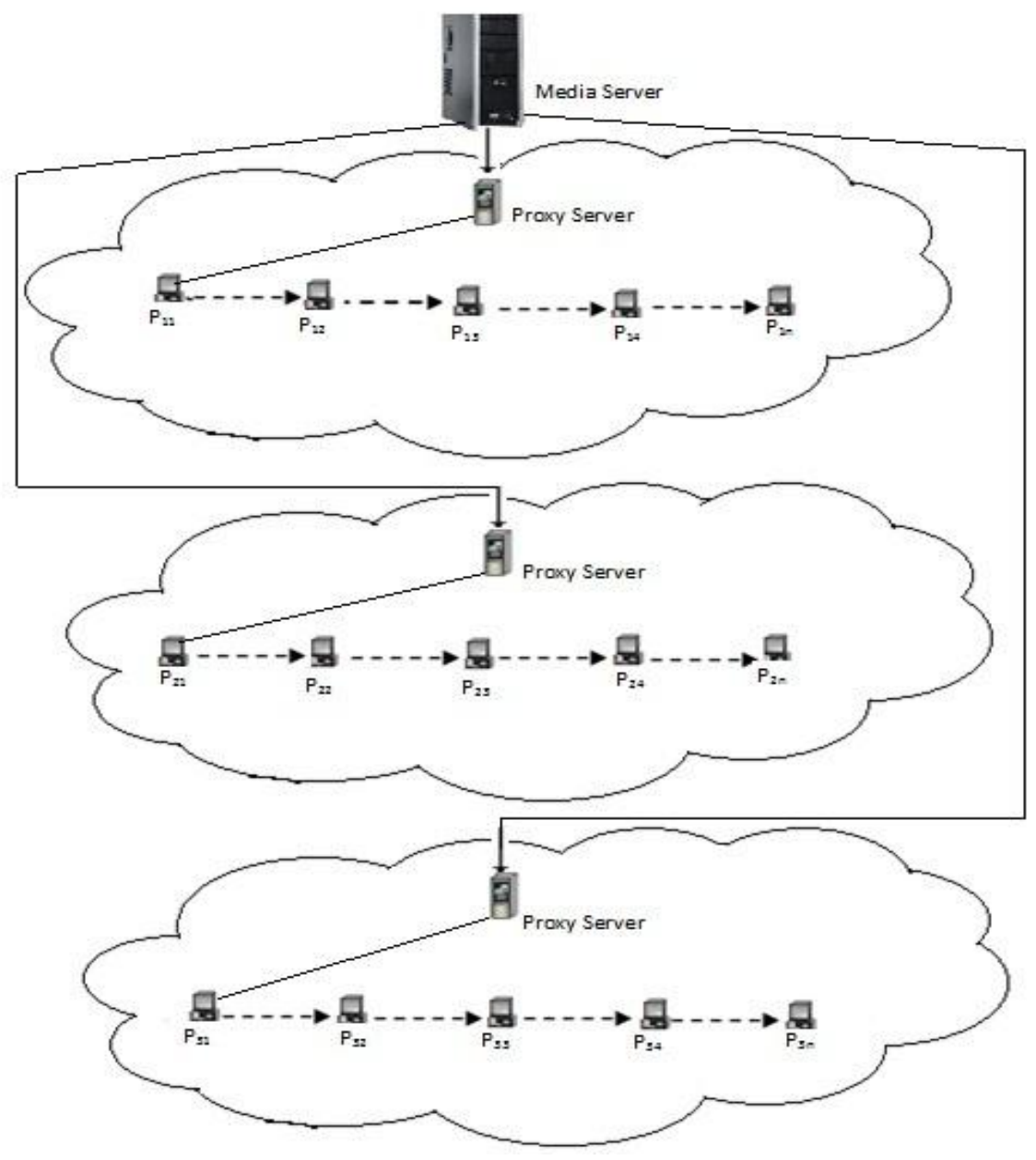

Figure 1 Peer to Peer VoD System Architecture

found in the server then the procedure is followed as if it is a first request from the cluster. Suppose, if another request from different cluster occurs for the same movie to the server. Then the server redirects the nearest proxy server of the requesting peer to transmit the video data file to the proxy server of the requesting peer to transmit the video data file to the proxy server of the requesting peer. Now instead of downloading the video data file from server to the nearest proxy server. The download happens from another proxy server which has the movie to the nearest proxy server of the requesting peer. Therefore, the same procedure is carried out for the transmission of movies among the clusters. If none of the proxy servers has the same movie then the server downloads to the nearest proxy server and then the proxy server transmits the movie to the requesting peer.

\section{CHAINING MECHANISM}

Now we proposed a mechanism for video transmission between the peers, which is known as V-Chaining mechanism. Here the time line of streaming session is taken as a reference to chain the peers. The time line is scale that units the inter arrival time between the peer. Let us consider the duration of movie $\mathrm{D}$ minutes. Then the factor in the timeline starts from 0 to $\mathrm{D}$ minutes. Let us assume that the chain has to be established between two peers that is $\mathrm{P}_{1}$ and $\mathrm{P}_{2}$. Let the inter arrival time be $\mathrm{AT}_{12}$ between the peers $\mathrm{P}_{1}$ and $\mathrm{P}_{2}$, where $\mathrm{AT}_{12}$ is the difference between the requests of peers $\mathrm{P} 1$ and $\mathrm{P}_{2}$. Let $\mathrm{B}_{12}$ be the bandwidth capacity between $\mathrm{P}_{1}$ and $\mathrm{P}_{2}$. Let $\mathrm{b}_{1}$ and $b_{2}$ be the buffer capacity of $P_{1}$ and $P_{2}$. Now the downloading time of $\mathrm{P} 1$ from source is calculated based on the size of the Movie $S$ and its reception rate $R_{1}$. The first condition is checked for chaining and is given as

$$
\mathrm{AT}_{12}<\frac{S}{R_{1}}
$$

If the condition in equation 1 satisfies, then a chain is established between $\mathrm{P}_{1}$ and $\mathrm{P}_{2}$. If the condition fails, then total playback rate $\operatorname{Pr}_{1}$ of $\mathrm{P}_{1}$ is calculated based on the video consumption rate. Now, the inter arrival time $\mathrm{AT}_{12}$ and the total playback rate of $\mathrm{P} 1$ is checked as second condition and is given as

$$
\mathrm{AT}_{12}<\frac{S}{P r_{1}}
$$


If the condition in equation 2 satisfies, then a chain is established between $\mathrm{P}_{1}$ and $\mathrm{P}_{2}$.If this condition fails, contains of buffer $b_{1}$ of $P_{1}$ is observed within streaming timeline. Now, the observation is estimated based on the transmission rate $r_{1}$ of $\mathrm{P}_{1}$ from source and the buffer $\mathrm{b}_{1}$ contains within the streaming timeline of the movie's duration $\mathrm{D}$. The interarrival time $\mathrm{AT}_{12}$ and the buffer $\mathrm{b}_{1}$ contain is checked as third condition and is given as

$$
\mathrm{AT}_{12}<\frac{b_{1}}{r_{1}}----------(3)
$$

If the condition in equation 3 satisfies, then a chain is established between $\mathrm{P}_{1}$ and $\mathrm{P}_{2}$.If none of the above condition is satisfied, then the video is obtained directly either from proxy server or multimedia server to peer $\mathrm{P}_{2}$. The above scenario of chaining between $\mathrm{P}_{1}$ and $\mathrm{P}_{2}$ can be generalized for any chaining between $\mathrm{P}_{1}$ and $\mathrm{P}_{2}$. Therefore; the generalized equations are derived as given in the below equations.

$$
\begin{aligned}
& \mathrm{AT}_{\mathrm{ij}}<\frac{S}{R_{i}}-----------(4) \\
& \mathrm{AT}_{\mathrm{ij}}<\frac{S}{P r_{i}}----------(5) \\
& \mathrm{AT}_{\mathrm{ij}}<\frac{b_{i}}{r_{i}}-----------(6)
\end{aligned}
$$

The generalized equations will be applied in the V-Chaining algorithm for $\mathrm{j}^{\text {th }}$ peer to establish a chain from $\mathrm{i}^{\text {th }}$ peer.

\section{V-CHAINING ALGORITHM}

\section{Nomenclature}

$\mathrm{AT}_{\mathrm{ij}}:$ : Inter arrival time between $\mathrm{i}^{\text {th }}$ and $\mathrm{j}^{\text {th }}$ peer, $\mathrm{S}::$ size of the

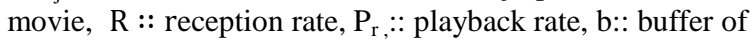
the peer, $r:$ : transmission rate of the movie

Step 1: Request arrival of $j^{\text {th }}$ peer

Step 2: Redirection from Server to $i^{\text {th }}$ peer

Step 3: Calculate $\mathrm{AT}_{\mathrm{ij}}=$ Arrival of $\mathrm{j}^{\text {th }}$ peer - arrival of $\mathrm{i}^{\text {th }}$ peer

Step 4: If $\mathrm{AT}_{\mathrm{ij}}<\frac{S}{R_{i}}$ then establish chain between $\mathrm{i}^{\text {th }}$ and $\mathrm{j}^{\text {th }}$

peer

Step 5: else if $\mathrm{AT}_{\mathrm{ij}}<\frac{S}{P r_{i}}$ then establish chain between $\mathrm{i}^{\text {th }}$ and $\mathrm{j}^{\text {th }}$ peer

Step 6: else if $\mathrm{AT}_{\mathrm{ij}}<\frac{b_{i}}{r_{i}}$ then establish chain between $\mathrm{i}^{\text {th }}$ and $\mathrm{j}^{\text {th }}$ peer

Step 7: else receive from server or from the proxy

\section{PERFORMANCE ANALYSIS}

In order to evaluate the performance of V-Chaining algorithm, we have considered bandwidth and buffer are the two very important parameters for analysis of the algorithm. The bandwidth utilization is measured at the server and as well as at the peer ends. The load on the server is measured in terms of bandwidth utilization at the server. The load at the server shall gradually decrease as the number of peers establishes the chains. Meanwhile, the load on the peer are collectively measured based on the bandwidth utilization at the peers. Since, the arrival of the peers follows a Poisson's process. Therefore, in our model, the probability density of peer's arrival is given as

$$
\mathrm{P}(\mathrm{t})=\lambda e^{-t}
$$

Where at time $t$ on the streaming time line an average of $\lambda$ peers arrive. The average bandwidth utilization at the server end is measured as

$$
B_{\text {Avg }}^{S}=\int_{0}^{T} D \lambda e^{-t}=D \lambda e^{-t}
$$

Therefore, the server load is calculated for an average of $\lambda$ peers and is given as

$$
S_{L=} \lambda B_{A v g}
$$

The average bandwidth utilization at the peers are measured as

$$
B_{A v g}^{P}=\int_{0}^{T} \frac{S . D}{r \lambda} e^{-\lambda t}=\frac{S . D}{r_{\lambda}} e^{-\lambda T}
$$

Therefore, the collective load on peers is calculated for an average of $\lambda$ peers is given as

$$
P_{L=} \lambda B_{A v g}^{P}
$$

The other important parameter of buffer is measured in terms of effective utilization of buffers at the peers. For each peer buffer utilization measured in terms of video contains in its storage for the duration of the movie $\mathrm{D}$ and the video consumption rate that is playback rate. Therefore, the average buffer utilization of buffer in a peer is given by

$$
b_{A v g}^{p}=\int_{0}^{D}\left(\frac{S}{r}-P_{r}\right) \mathrm{dt}
$$

The average buffer utilization of buffers in all the peers is given by

$$
b_{A v g}^{P_{s}}=\lambda b_{A v g}^{P}
$$

\section{SIMULATION AND RESULTS}

In this section, we use simulation to evaluate the performance of the VoD system. Matlab software was used for the purpose of the simulation. The topology used in our simulation consists of a single media server and 5 cluster based network. Each cluster constitutes a proxy server and 500 peers which includes the seed peers and the non seed peers. The media server consists of bandwidth with a capacity of $100 \mathrm{Mbps}$ and buffer capacity of $5000 \mathrm{MB}$. The proxy server bandwidth capacity is ranging from $30 \mathrm{Mbps}$ to $50 \mathrm{Mbps}$ and buffer capacity ranging from $500 \mathrm{MB}$ to $1000 \mathrm{MB}$. Each seed peer has a bandwidth capacity of $5 \mathrm{MB}$ to $10 \mathrm{MB}$ and buffer capacity of $1000 \mathrm{MB}$. The non seed peer has a bandwidth capacity of less than $5 \mathrm{MB}$ and buffer capacity of less than $800 \mathrm{MB}$. The proxy server maintains a database of currently streamed/streaming movies and a list of seed peers. The media server contains the information about the movies such as index, size, duration, popularity etc., and this server also maintains two separate files for each of the movie. One file contains the segmented parts of the movie and other file contains the frames of the scene boundary locations. The average duration of each movie is 7200 seconds. The total number of movies requested in a cluster is less than 300 requests during the peak duration. It follows a Poisson request rate with mean $\lambda=50$ during the normal durations. The average normal playback rate of each movie is $2 \mathrm{MB}$. The popularity of each movie selects according into Zipf's Law. The operations are executed on a timeline between 500 seconds and 5000 minutes in a cluster and follows the Poisson request rate with mean $\lambda^{\prime}=50$. This simulation model is executed for several times and the result shown is an average of all simulation trails carried out in all 5 clusters. We compared our analytical results with our simulation. Our simulation program assumed that request arrivals for a particular video were distributed according to a Poisson law and simulated requests for a single two-hour video of MPEG-2 format. We measured the average server bandwidth at request arrival rates varying between one and Five thousand requests per minutes. We did not consider higher arrival rates as they seemed unrealistic. Each simulation run involved at least 5,000 arrivals over a simulated time period of at least 5000 minutes. The simulation model is evaluated for several times. The result shown is an average of all simulation trials carried out in all clusters. We simulated different chaining approaches like standard, advanced, optimal, and accelerated with our proposed VChaining mechanism. Figure 2 shows the server bandwidth with the number of requests. We have observed that, As in 
case of our proposed V-Chaining mechanism, consumption of bandwidth is $22 \mathrm{Mbps}$, which is very less compared all the above approaches because When the requests of movie from the peers arrive to the system, it checks the difference of interarrival time of the new peer and other peers of the existing chain, which is active is less than the duration of movie D minutes, The various conditions with which the chaining occurs will be clearly disucussed in section in section 4. For this requests of peers to be served immediately from the active chain and also add them to the currently available existing active chain due to this consumption of server bandwidth is $22 \mathrm{Mbps}$ in our proposed approach. Accordingly numer of peers chain among with other peers. As the chain fails the peer stream the video from the server. The server bandwidth measured based on the inter arrival of requests, initially less bandwidth consumption in all the approaches as the time increases the bandwidth also increases up to 80 Mbps consumption in case of standard chaining. Also we observed in advanced, optimal and accelerated chaining bandwidth consumption up to $75 \mathrm{Mbps}$, $55 \mathrm{Mbps}$ and 40 Mbps respectively.

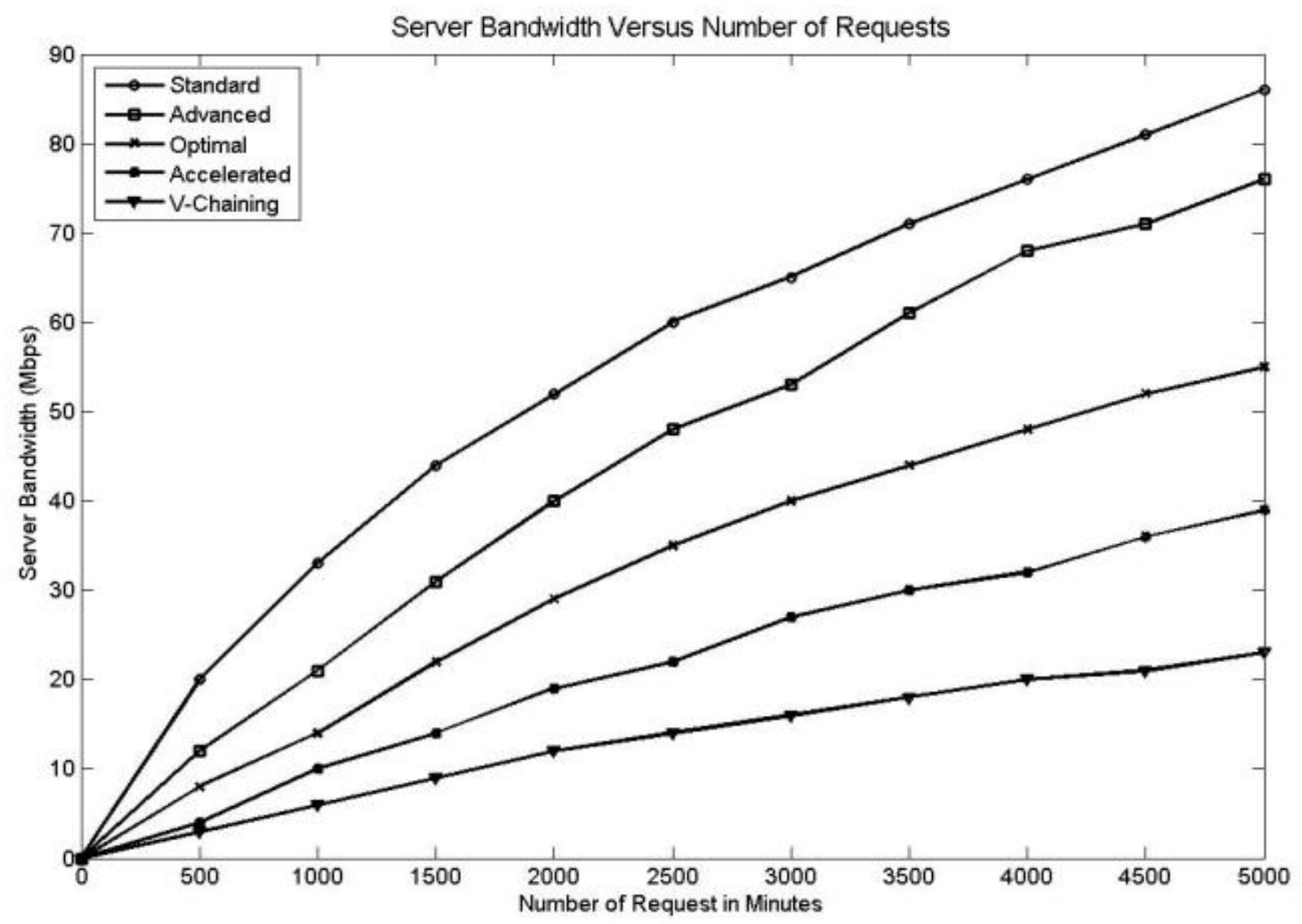

Figure 2 Server bandwidth versus Number of requests

Figure 3 shows the load on the server versus number of requests. When the requests of videos from the peers arrive to the system, it checks the difference of interarrival time of the new peer and other peers of the existing chain, which is active is less than the duration of movie D minutes, The various conditions with which the chaining occurs will be clearly disucussed in section in section 4. For this requests of peers to be served immediately from the active chain and also add them to the currently available existing active chain. We measured the load on the server in terms of bandwidth utilization at the server. As the inter arrival of requests of peers for the movie varies between 0 to 5000 minutes the load on the server is $28 \%$ in case of our proposed approach VChaining, which is less compared to all the existing chaining approaches, because the requested video streams is available in the nearby peers, accordingly the requested peer chain among the peers establishes the chaining. As in case of other approaches, the peers are chaining, if the condition discussed in section 4 will not satisfied, then the video streams are directly streamed from the server. Hence the load on the server gradually increases. Therefore we observed in standard chaining an average of $92 \%$ load on the server. In case of advanced optimal and accelerated chaining the load on the server will be $80 \%, 60 \%$ and $42 \%$ respectively.

Figure 4 shows the peer bandwidth versus number of requests. We have observed the peer bandwidth utilization based on the number of inter-arrival of requests of movie by the peer. When the requests of videos from the peers arrive to the proxy server,it redirects to the requested peer movie id is availabe in the near by peers, accordingly the peer checks the Condition, which is discussed in section 4, if the condition is Satisfied the peer upload the video streams through uplink to the requested peer chain among the peers establishes the chaining. Hence, our proposed V-Chaining mechanism 10 to $20 \mathrm{Mbps}$ of the peer bandwidth utilization, which is less compared to all the existing approaches. As in existing approaches the peers are fails in satisfy the condition discussed in section 4 . 
Therefore the bandwidth utilization in peers in case of standard, advanced, optimal and accelerated chaining is 90 to $100 \mathrm{Mbps}, 70$ to $80 \mathrm{Mbps}$, 50to $60 \mathrm{Mbps}$ and 30 to $40 \mathrm{Mbps}$ respectively. Figure 5 shows the collective peer bandwidth versus number of requests. We observed

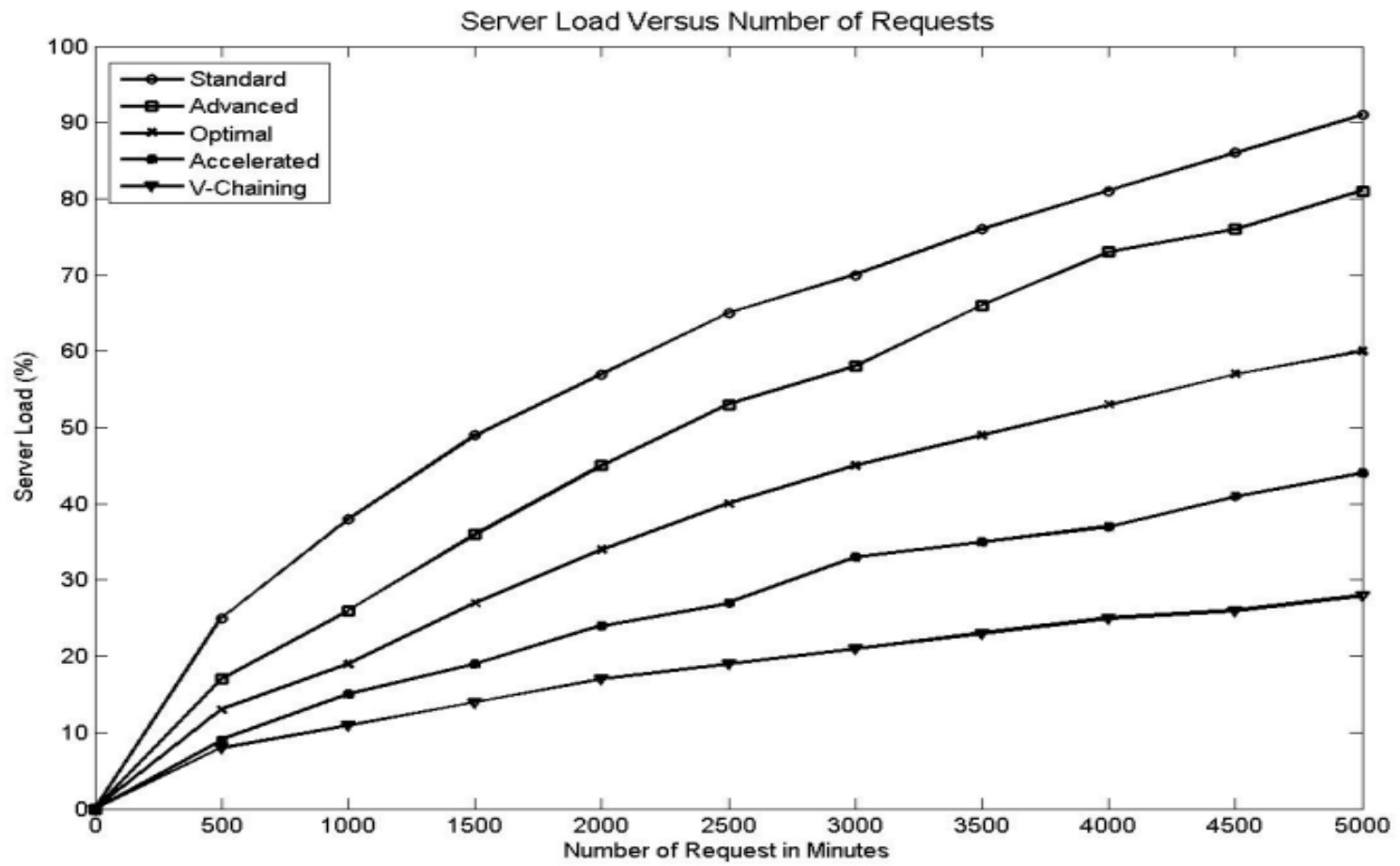

Figure 3 Load on the Server versus Number of requests

the peer bandwidth utilization based on the number of interarrival of requests of movie by the peer. As we used the time line between 0 to5000 minutes, the load on the peers is collectively measured based on the bandwidth utilization of peer, which is clearly discussed in section 6 . As we observed from the simulation, the collective peer utilization is 90 to 105
Mbps in standard chaining. In case of advanced, optimal and accelerated chaining of bandwidth utilization is 70 to 85 Mbps, 50 to $65 \mathrm{Mbps}$ and 30 to $45 \mathrm{Mbps}$ respectively. In case of our proposed V-Chaining mechanism the peer bandwidth utilization is 15 to $25 \mathrm{Mbps}$, which is less compared to all the existing approaches.

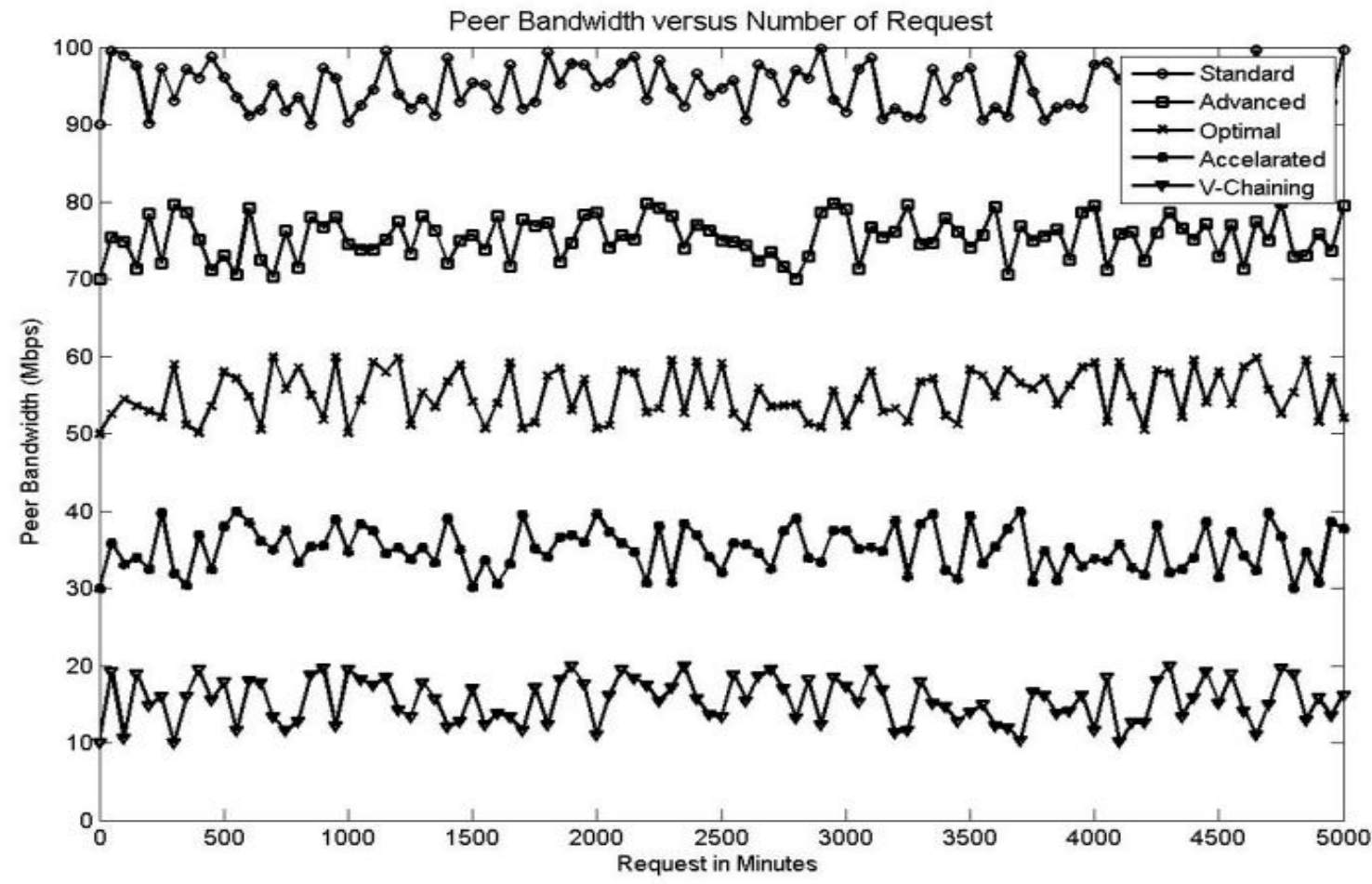

Figure 4 Peer bandwidth versus Number of requests. 


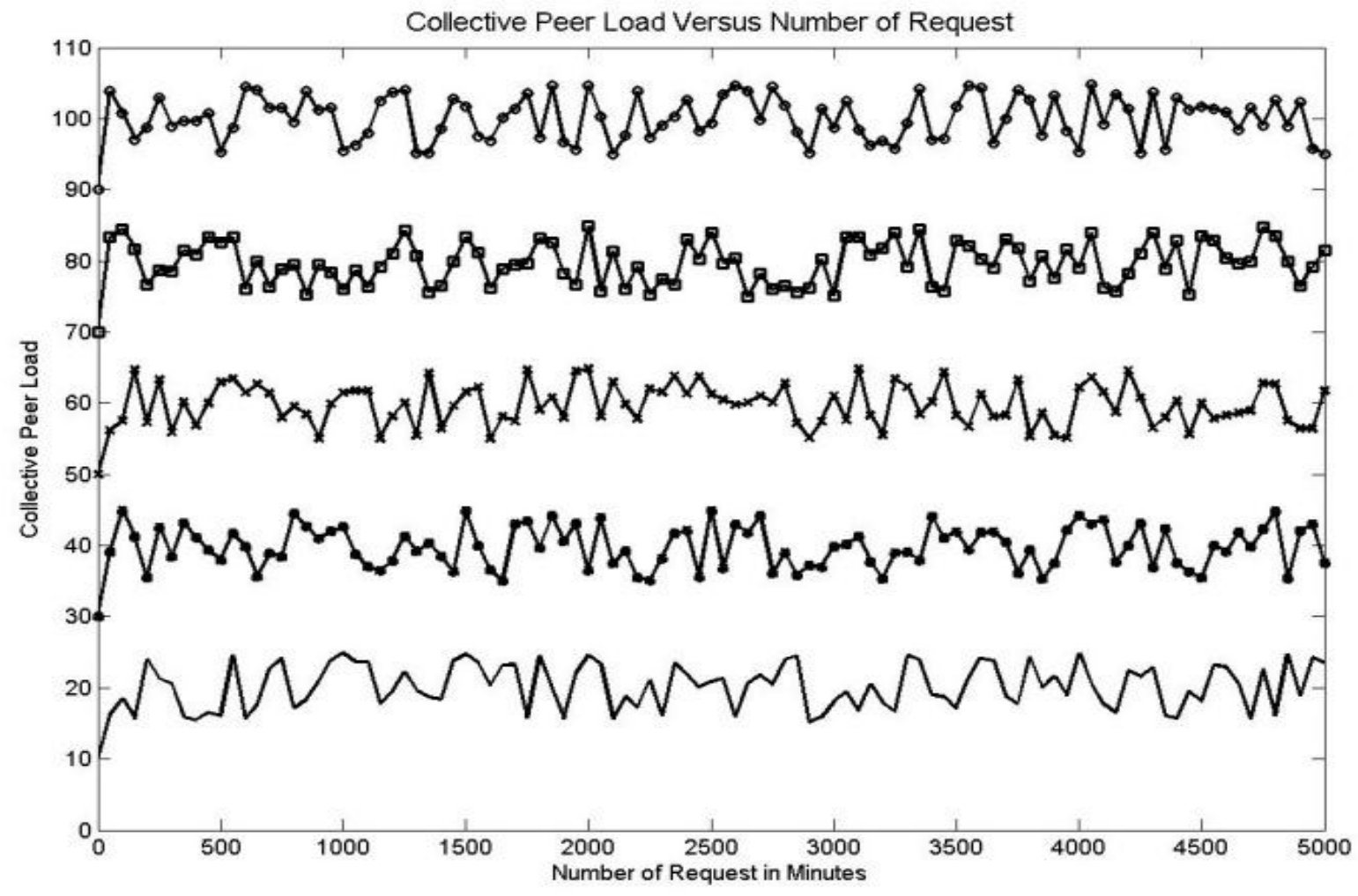

Figure 5 Collective peer bandwidth versus Number of requests

Figure 6 shows the average buffer utilization in each peer versus number of requests of the movie and play back rate. We have observed that, the buffer utilization for each is measured in terms of video contains in its storage for the duration of the movie $\mathrm{D}$ and the video consumption rate that is playback rate, which is clearly discussed in section 6 . We have observed that in case of our proposed V-Chaining mechanism it checks the buffer as discussed the condition in section 4, an average of 300 to $600 \mathrm{MB}$ of buffer utilization in each of peer for its chaining among the peers. Accordingly we have measured from the simulation, in case of standard, advanced, optimal and accelerated chaining approaches approximately utilize the buffer as $5000 \mathrm{MB}, 3000 \mathrm{MB}$, $2000 \mathrm{MB}$ and $1000 \mathrm{MB}$ respectively.

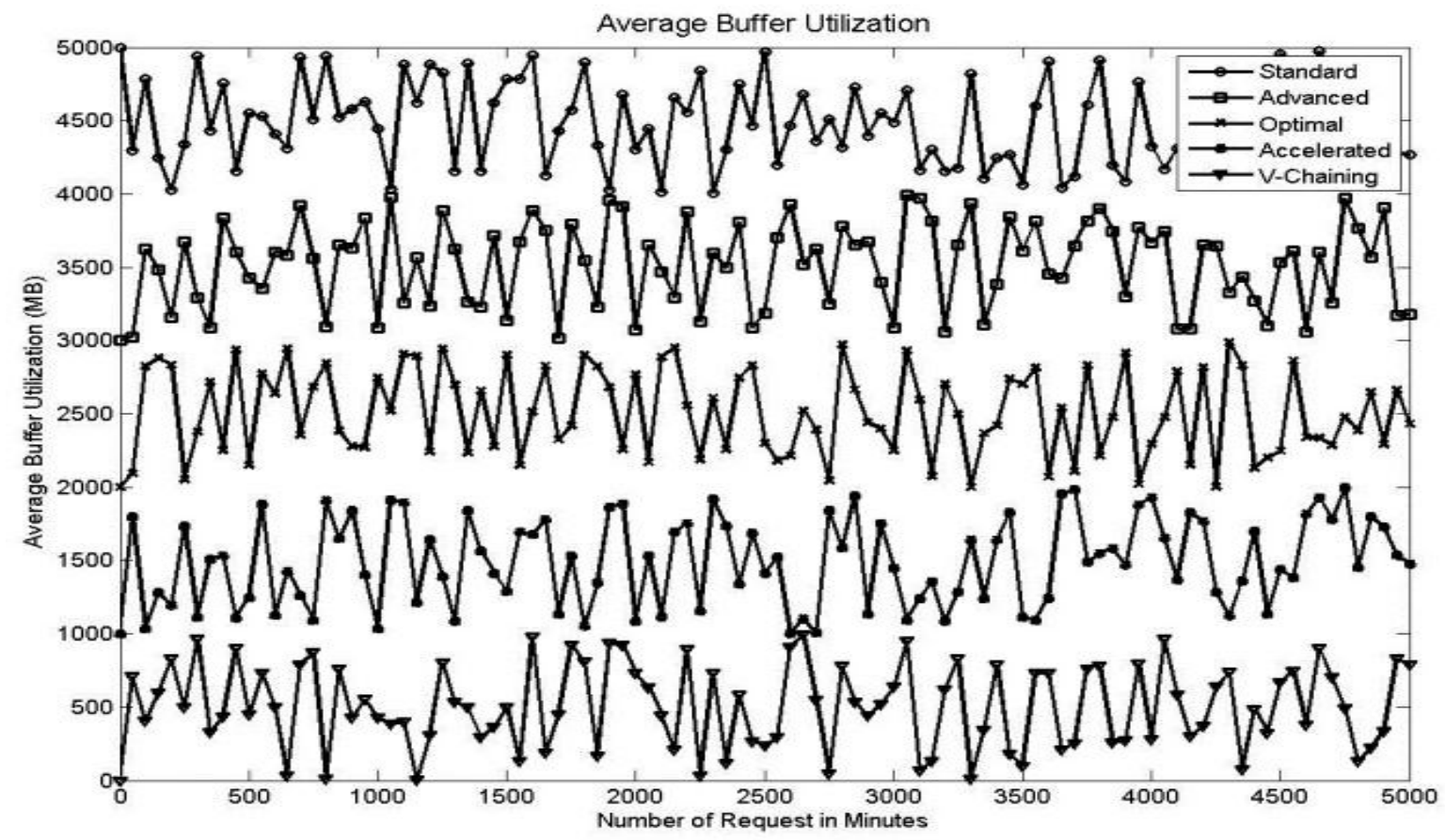

Figure 6 Average buffer utilization in each peer 
Figure 7 shows the average buffer utilization in all peers versus number of requests of the movie in a time line varies between 0 to 5000 minutes. We have observed that, the buffer utilization for all peers is measured in terms of video contains in its storage for the duration of the movie D and the video consumption rate that is playback rate, which is clearly discussed in section 6.we have observed from the simulation, in case of our proposed V-Chaining an average of 400 to 1000 MB of buffer utilization in each of peer for its chaining among the peers. We have observed that, the buffer utilization in case of standard, advanced, optimal and accelerated chaining approaches approximately evaluated as 4200 to $5200 \mathrm{MB}$, 3000 to $4200 \mathrm{MB}, 2000 \mathrm{MB}$ and $3200 \mathrm{MB}$ respectively.

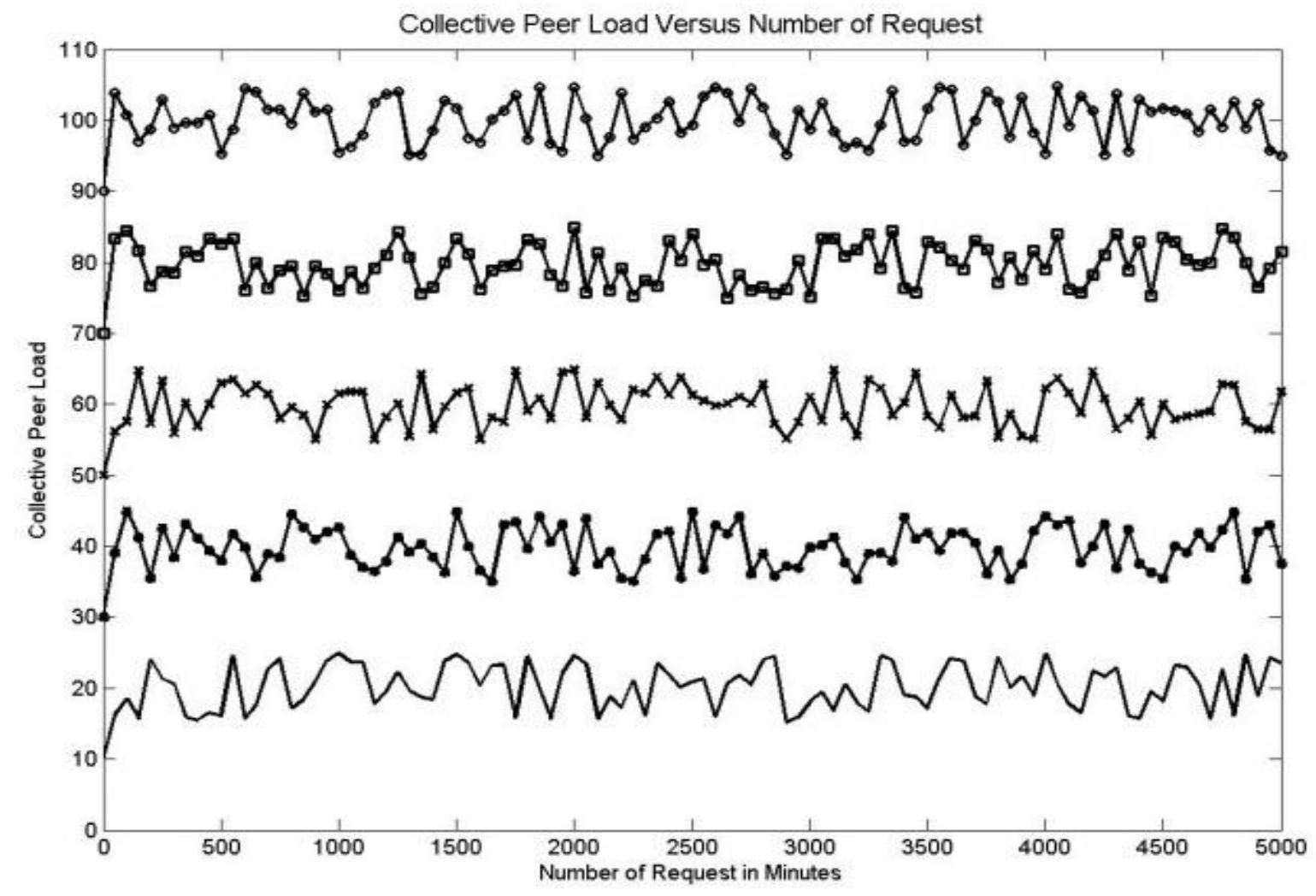

Figure 7 Average buffer utilization in all peers versus Number of requests

\section{CONCLUSION}

Chaining mechanism reduces the load on the server, which is measured the system resources like bandwidth and buffer. In this paper we have evaluate the performance of peer to peer video on demand system using V-Chaining Mechanism. We have proposed V-Chaining algorithm on VoD system architecture for the transmission of video streams to the peers that improves the performance compared with existing approaches such as standard, advanced, optimal and accelerated chaining. In comparison to it, we have also considered some of the existing approaches that use chaining mechanism for the transmission of video streams for the video on demand system. The drawback of these existing approaches not efficiently utilized the system resources like bandwidth and buffer. Hence we have proposed V-Chaining mechanism that enhances the performance of the chaining mechanism in terms of bandwidth, buffer in both server and peer load. Also we have derived an analytical model for different performance metrics for our proposed mechanism and the same is simulated to have fairer comparisons between the existing mechanisms with our V-Chaining mechanism. The simulation results shows that our proposed mechanism fairs in all aspects of performance metrics than the existing approaches and also shows that better utilization of bandwidth and buffer in the server as well as peers as the number of peers increases. Further enhancement of this paper is to evaluate fault tolerance; when chaining fails among the peers under various extreme circumstances.

\section{REFERENCES}

[1] X. Zhang, J. Liu, B. Li, and T.S.P. Yum 2005, 'Donet/Coolstreaming: A Datadriven Overlay Network for Live Media Streaming', Proc. IEEE INFOCOM.

[2] Y. Huang, T.T.J. Fu, D.M. Chiu, J.C.S. Lui, and C. Huang 2008,'Challenges, Design, and Analysis of a Large-Scale P2P VoDSystem', Proc. ACM SIGCOMM.

[3] Lee J. Y. B. 1998,'“Parallel Video Servers': A Tutorial, IEEE Multimedia, vol.5(2), pp.20-28.

[4] Xuguang Lan, Nanning Zheng, Jianruxue, Weike Chen, Bin Wang, and Wenma 2008, 'Manageable Peer-toPeer Architecture for Video-on-Demand', IEEE International Symposium on Parallel and Distributed Processing.

[5] Nader F. Mir,Savitha Ravikrishnan and Meera M. Nataraja 2011,'Analysis of Reliable and Scalable VideoOn-Demand Networks"ICN 2011 : The Tenth International Conference on Networks,pp 430 to 435 :St. Maarten, The Netherlands Antillesrom.

[6] S. Sheu, K. A. Hua, and W. Tavanapong 1997,'Chaining: a generalized batching technique for video-on-demand systems', Proc. IEEE International Conference on 
Multimedia Computing and Systems (ICMS '97),

[7] J. K. Chen and J. L. C. Wu 1999,'Adaptive chaining scheme for distributed VOD applications', IEEE Trans. Broadcast., vol. 45, no. 2, pp. 215-224

[8] Bing Wang, S. Sen, Micah Adler, and Don Towsley 2004,'Optimal Proxy Cache Allocation for Efficient Streaming Media Distribution', IEEE Trans. Multimedia, vol. 6, no. 2.

[9] G. Barlas, B. Veeravalli 2005,'Optimized Distributed Delivery of Continuous-Media Documents over Unreliable Communication Links', IEEE Trans. parallel and distributed systems, vol. 16, no. 10.

[10] Frederic Thouin and Mark Coates, 2007, McGill University, 'Video-on- Demand Networks: design Approaches and Future Challenges', IEEE Network.

[11] K. A. Hua, S. Sheu, and J. Z. Wang 1997,'Earthworm: a network memory management technique for large-scale distributed multimedia applications', in Proc. IEEE INFOCOM '97, vol. 3, Kobe, Japan, pp. 990-997.

[12] T.-C. Su, S.-Y. Huang, C.-L. Chan and J.-S. Wang 2005,'Optimal chaining scheme for video-on-demand applications on collaborative networks', IEEE Transactions on Multimedia, 7(5): 972-980
Berlin,Germany, pp. 110-117

[13] J.-F Pâris, A. Amer and Darrell D. E. Long 2011, 'Accelerated chaining: a better way to harness peer power in video-on-demand applications', Proc. 26th ACM Symposium on Applied Computing (SAC 2011), Taichung, Taiwan, in press.

[14] F. Lin, C. Zheng, X. Wang, X. Xue 2007, 'ACVoD: A peer-to-peer based video-on-demand scheme in broadband residential access networks', International Journal of Ad Hoc and Ubiquitous Computing, 2(4)4.

[15] T.-C. Su, S.-Y. Huang, C.-L. Chan and J.-S. Wang 2002 , 'Optimal chaining and implementation for large scale multimedia streaming', Proc. 2002 IEEE International Conference on Multimedia and Expo (ICME 2002), Lausanne, Switzerland, Vol.1,August, pp. 385-388.

[16] J.-F Pâris 2005, 'A cooperative distribution protocol for video-on-demand', Proc. 6th Mexican International Conference on Computer Science, Puebla, Mexico, pp. 240-246.

[17] On G., Zink M., Liepert M., Griwodz C., Schmitt J.B., and Steinmetzr 2001, 'Replication for a Distributed Multimedia System', In Proceedings of the Eighth International Conference on Parallel and Distributed Systems, pp.37-42 